\title{
Mutation of fibulin-1 causes a novel syndrome involving the central nervous system and connective tissues
}

\author{
Saeed Bohlega ${ }^{1}$, Huda Al-Ajlan² and Amr Al-Saif ${ }^{\star, 2,3}$ \\ Fibulin-1 is an extracellular matrix protein that has an important role in the structure of elastic fibers and basement \\ membranes of various tissues. Using homozygosity mapping and exome sequencing, we discovered a missense mutation, \\ p.(Cys397Phe), in fibulin-1 in three patients from a consanguineous family presented with a novel syndrome of syndactyly, \\ undescended testes, delayed motor milestones, mental retardation and signs of brain atrophy. The mutation discovered \\ segregated with the phenotype and was not found in $\mathbf{3 7 4}$ population-matched alleles. The affected cysteine is highly \\ conserved across vertebrates and its mutation is predicted to abolish a disulfide bond that defines the tertiary structure of \\ fibulin-1. Our findings emphasize the crucial role fibulin-1 has in development of the central nervous system and various \\ connective tissues.
}

European Journal of Human Genetics (2014) 22, 640-643; doi:10.1038/ejhg.2013.210; published online 2 October 2013

Keywords: FBLN1; fibulins; homozygosity mapping; exome sequencing; neurodegenerative disease

\section{INTRODUCTION}

Fibulins are extracellular glycoproteins secreted in elastic fibers and basement membranes of various tissues where they interact with several extracellular matrix (ECM) components. They have an important role in the assembly and stabilization of supramolecular ECM complexes. The fibulin family is characterized by a tandem repeat of epidermal growth factor-like modules and a unique C-terminal fibulin-type module. ${ }^{1}$

Fibulin-1 (the first discovered member of the fibulin family) is a highly conserved protein across metazoa. ${ }^{2}$ It is encoded by FBLN1 gene, which has four isoforms that differ in their ultimate exons. It is expressed in various tissues such as vessel walls, cartilages, skin and lung. During development, it is prominently expressed in the neural crest cells, and is implicated in the morphogenesis of the cranial neural crest-derived structures. It is implicated in tissue organogenesis in developing myotomes, endocardial cusion and digits of the developing limbs. ${ }^{1,3}$ A translocation involving the last exon of isoform $D, t(12 ; 22)(\mathrm{p} 11.2 ; \mathrm{q} 13.3)$ has been associated with polysyndactyly. ${ }^{4}$ Authors proposed that haploinsufficiency of isoform $\mathrm{D}$ was responsible for the limb malformation they reported. Using a combination of homozygosity mapping and exome sequencing, we report the first point mutation of fibulin-1 and confirm its segregation in a novel autosomal recessive syndrome affecting the development of the central nervous system and connective tissues.

\section{MATERIALS AND METHODS}

Patients and DNA samples

Three affected individuals together with their parents and siblings were recruited for the study at King Faisal Specialist Hospital and Research Center.
Informed consents were obtained from all participating individuals, and $5-10 \mathrm{ml}$ of venous blood was drawn. DNA was extracted from lymphocytes using Puregene DNA extraction kit (Qiagen, Valencia, CA, USA).

\section{Homozygosity mapping}

Cytogenetics Whole-Genome $2.7 \mathrm{M}$ Arrays (Affymetrix, Santa Clara, CA, USA) were used to genotype single-nucleotide polymorphisms (SNPs) in all affected individuals according to the manufacturer's protocol. Homozygosity analysis was performed using Chromosome Analysis Suite (Affymetrix). Shared homozygosity regions between the three affected individuals (II:1, II:2 and II:6) were exported to be used as a filter for exome sequence variants.

\section{Exome sequencing}

A DNA sample from one of the affected individuals (II:2) was used for exome sequencing on SOLiD 4 instrument (Applied Biosystems, Foster City, CA, USA) using Agilent (Santa Clara, CA, USA) SureSelect Human All Exon 50 MB kit with $50 \times$ average coverage. Sequences were aligned using the NovoAlign aligner (NovoCraft, Selangor, Malaysia) and variants were called using The Genome Analysis Toolkit (Broad Institute, Cambridge, MA, USA). Cut-off values for quality scores were: $\mathrm{QUAL}>50$ and $\mathrm{DQ}>8$.

\section{PCR and Sanger sequencing}

The segment surrounding the c.1190G $>$ T mutation was PCR amplified for all family members. Amplicons were then sequenced using BigDye Terminator sequencing kit (Applied Biosystems) and run on an ABI3130xl genetic analyzer (Applied Biosystems). Sequencher software (Gene Codes Corporation, Ann Arbor, MI, USA) was used to align sequence data to reference sequence (GenBank:NM_006486).

The detected variant has been submitted to ClinVar Database (URL: http:// www.ncbi.nlm.nih.gov/clinvar/).

${ }^{1}$ Department of Neurosciences, King Faisal Specialist Hospital and Research Center, Riyadh, Saudi Arabia; ${ }^{2}$ Department of Genetics, King Faisal Specialist Hospital and Research Center, Riyadh, Saudi Arabia; ${ }^{2}$ Laboratory of Neurogenetics, National Institutes of Health, Bethesda, MD, USA

${ }^{*}$ Correspondence: Dr A Al-Saif, Laboratory of Neurogenetics, National Institutes of Health, 35 Convent Dr. Rm 1a1015 Bethesda, MD 20892 , USA. Tel: +1 3014516081 Fax: +1 301451 7295; E-mail: amr.al-saif@nih.gov

Received 8 May 2013; revised 1 August 2013; accepted 16 August 2013; published online 2 October 2013 


\section{RESULTS}

\section{Clinical presentation}

Three affected children of second-cousin parents (Figure 1) presented with signs of limb malformation and/or undescended testes at birth and later showed abnormal developmental milestones.

Individual II:1. Individual II:1 presented with syndactyly in her left hand at birth. She had delayed crawling and walked independently at the age of 18 months. She was diagnosed with infantile spasm with corresponding EEG changes at the age of 2 years. The spasm resolved with corticosteroid therapy. She also suffered from bilateral hip dislocation and required bilateral hip replacement. At age 23, she started to have stiffness and numbness in the left leg. MRI of the brain showed cortical atrophy and few scattered white matter lesions (Figure 2a). Cervical spine MRI showed compression at C3 and C4 levels, and syringomyelia at lower thoracic region (Figure $2 \mathrm{~b}$ and $\mathrm{c}$ ). She underwent cervical spine decompression surgery successfully but remained with mild leg spasticity. Cognitive milestones were normal. She had normal vision.

Individual II:2. Individual II:2 presented with bilateral foot syndactyly and undescended testes at birth. He suffered from static encephalopathy and could not read or write. Speech was spastic and dysarthric with pseudobulbar signs. He had dystonic and spastic posturing of arms and legs with impaired postural reflexes and suffered from moderate mental retardation. Upon ophthalmoscopic examination, he was found to have macular degeneration evidenced by subretinal fibrosis and choroidal neovascularization. Brain MRI

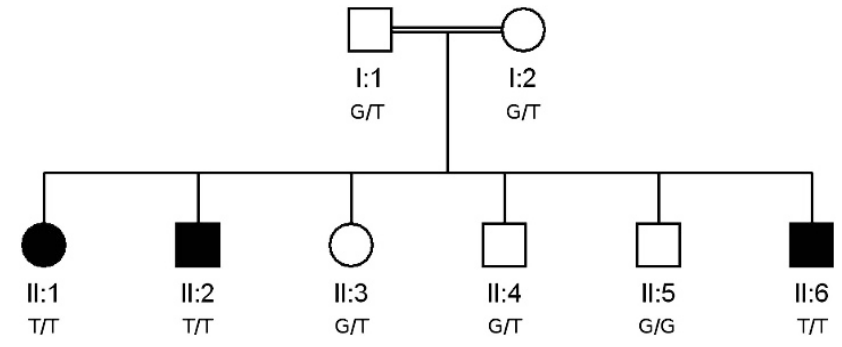

Figure 1 Pedigree of the family. Affectation status and genotypes for the c.1190G > T mutation are indicated.
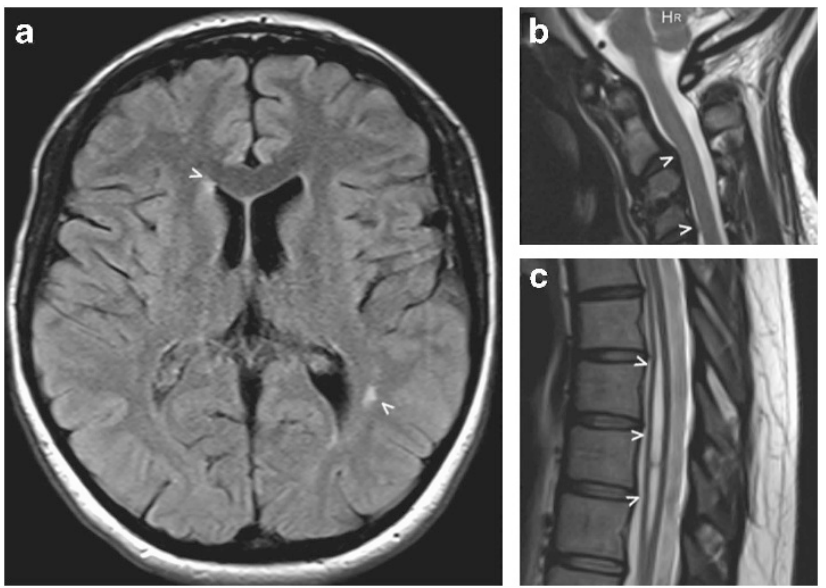

Figure 2 Brain and spinal abnormalities in patients. MRI scans for patient (II:1) show bilateral temporal lobe atrophy and white matter lesions (arrowheads; a), compression of cervical spine (b) and syringomyelia (c). showed generalized hemispheric atrophy. Brain MR angiography showed no abnormailities in brain vessels. Echocardiography did not show changes in the heart or heart great vessels.

Individual II:6. Individual II:6 presented with bilateral undescended testes with no syndactyly, but suffered from delayed speech and motor milestones. He was able to walk with walker but he required bilateral tendotomies and release of the thigh adductor muscles to relieve spasticity. At the age of 16 years, he was ambulatory on powered wheelchair. He had dysarthria, drooling and pseudobulbar signs. $\mathrm{He}$ suffered from moderate mental retardation. MRI of the brain revealed moderate brain atrophy. He showed no signs of macular degeneration.

\section{Detection of a point mutation in FBLN1 using homozygosity} mapping and exome sequencing

Based on the autosomal recessive inheritance pattern, rarity of the syndrome and the fact that parents are second cousins, we hypothesized the existence of a founder mutation in a common ancestry of both parents. Therefore, we looked for autozygous regions within affected individual genomes using high-density whole-genome SNP genotyping arrays. Results were scanned for segments of homozygosity shared by the three affected individuals (where the potential mutation is expected). We found that these segments accounted for about $6 \%$ of the genome $(\sim 180 \mathrm{Mbp})$. Next, we sequenced the exome of one of the affected individuals (II:2) and used the following criteria to filter the 32000 + variants detected by exome sequencing: (1) within shared homozygosity segments, (2) not in 1000 genome project database, (3) non-synonymous coding and (4) homozygous. These criteria successfully filtered out all but a single variant (c.1190G $>$ T) on exon 10 of FBLN1 gene located on chromosome 22. This variant results in the substitution of a cysteine residue at position 397 of fibulin-1 protein by phenylalanine, p.(Cys397Phe). We confirmed this variant using Sanger sequencing (Figure 3a) and genotyped family members for this variant. Genotypes perfectly segregated with the phenotype (Figure 1). In addition, to rule out a possible SNP, we sequenced 374 population-matched alleles and all were negative for this variant.

Nature of the mutation and predicted effect on protein structure The protein encoded by FBLN1 (fibulin-1) is an ECM protein characterized by nine tandem EGF-like domains that are present in many extracellular proteins and have crucial role in their diverse functions. These domains are 40-amino-acids long and characterized by six conserved cysteine residues that form three disulfide bonds, and determine the tertiary structure of the domain. ${ }^{5}$ Many EGF-like domains need to bind calcium for their biological function. The mutation we detected in fibulin-1 affects the sixth cysteine residue of the calcium-binding EGF-like domain number five. This cysteine is highly conserved across vertebrates (Figure $3 b$ ), and its substitution is predicted to significantly affect the structure of the fifth EGF-like domain (a shared domain between the four fibulin-1 isoforms), as it abolishes the disulfide bond between the fifth and sixth cysteine residues of this domain (Figures $3 \mathrm{c}$ and 4 ). As the function of fibulin-1 is not well understood, it is difficult to determine the functional effect of this mutation; however, based on the role this specific cysteine residue has in determining protein structure and its high level of conservation, it is expected to be detrimental to the function of fibulin-1. This detrimental effect is further supported by the high score (1.0) for this mutation obtained from the PolyPhen-2 algorithm. ${ }^{6}$ 
a
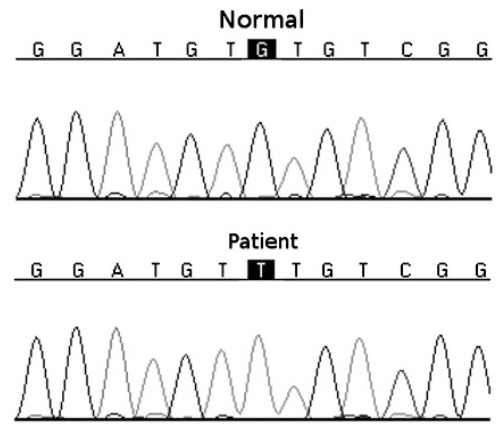

b

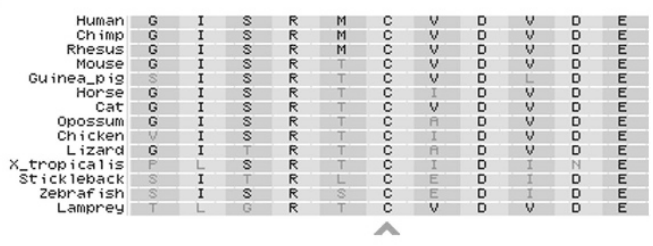

C

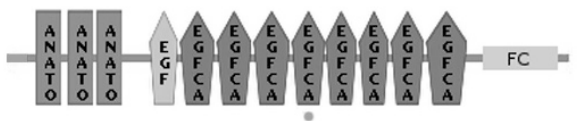

Figure 3 Location of the mutation and conservation of the affected residue. (a) Sequence chromatograms of the c.1190G $>T$ mutation compared with normal control. (b) The affected cysteine is conserved across vertebrates as shown in the conservation track of UCSC genome browser. (c) General structure of fibulin-1 (Source: Human Protein Reference Database, with modification) ${ }^{14}$ showing the location of affected domain (red dot). ANATO, anaphylatoxin domain; EGF, epidermal growth factor-like domain; EGFCA, calcium-binding EGF; FC, C-terminal fibulin-type module. The full colour version of this figure is available at European Journal of Human Genetics online.

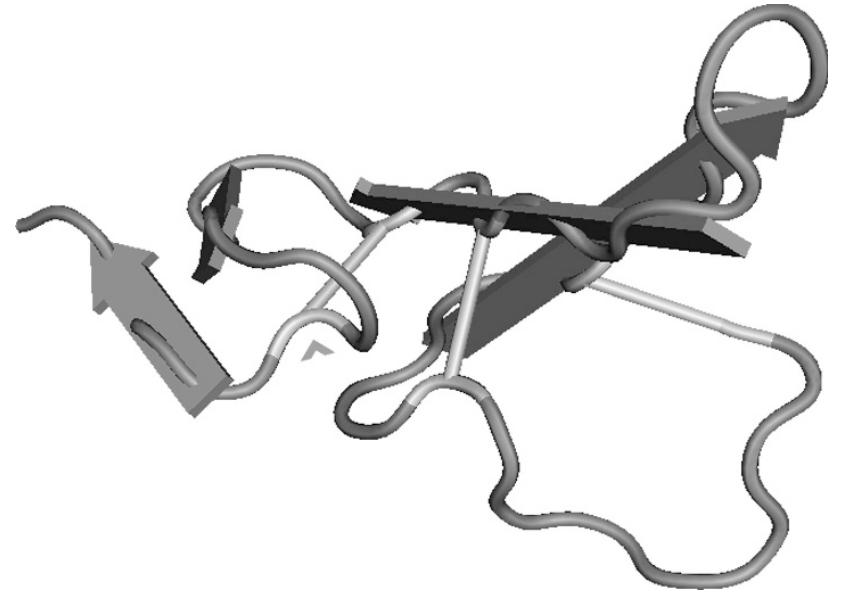

Figure 4 Effect of the mutation on the EGF-like domain structure. A 3D model showing the tertiary structure of human EGF with three disulfide bonds (yellow lines) and the corresponding location of the mutated cysteine (arrowhead). (Source: NCBI Molecular Modeling Database, MMDB ID: 17704). ${ }^{15,16}$ The full colour version of this figure is available at European Journal of Human Genetics online.

\section{DISCUSSION}

Several hereditary disorders have been associated with mutations in fibulin family proteins. Examples are cutis laxa with fibulin-4 and fibulin-5; age-related macular degeneration with fibulin-3, fibulin-5 and fibulin-6; and aortic aneurysm and arachnodactyly with fibulin-4. ${ }^{7-11}$ Here we have shown that a mutation affecting a highly conserved cysteine residue in fibulin-1 leads to multiple overlapping phenotypes in three affected siblings, including syndactyly, cryptorchidism, delayed motor milestones, pseudobulbar signs, mental retardation and evidence of brain atrophy, and compression of spinal cord in MRI scans. These phenotypes involve multiple systems and (with the exception of macular degeneration in individual II:2) are unique to fibulin-1, which indicates a specific role for fibulin-1 in ECM assembly and function. The early age of onset indicates the importance of fibulin-1 in intrauterine development of the brain and connective tissues. One of the associated symptoms, syndactyly, has been reported previously in a family with translocation involving the $\mathrm{D}$ isoform, it was the only manifestation in that family and was dominantly transmitted. ${ }^{4}$ However, none of the parents or carriers in our case showed syndactyly. This could be explained by dominant negative effect of the translocation mutation compared with the point mutation reported here, which is more likely consistent with loss of function effect. Also, these data may indicate a specific role of isoform $\mathrm{D}$ in limb development.

Although little is known about the function of the EGF-like domains of fibulins, the fifth EGF-like domain of fibulin-1 is a calcium-binding domain that has been shown to have a role in the localization of the protein and its self-interaction, as well as interaction with other proteins such as hemicentin. ${ }^{12,13}$

Our approach of homozygosity mapping and exome sequencing showed how robust this method is in discovering the genetic defect in families with high consanguinity. Limiting our screening to the shared homozygosity regions has helped us rule out $>99 \%$ of detected variants. Further data filtering for only coding homozygous variants lead us to a single variant that was later confirmed by Sanger sequencing method.

In conclusion, we used the combined approach of homozygosity mapping and exome sequencing to discover the genetic defect underlying a novel syndrome affecting the central nervous system, connective and other tissues. We showed how this approach was successful to pinpoint a single variant. We also showed that the discovered mutation affects a highly conserved residue and potentially affects the tertiary structure of fibulin-1.

\section{CONFLICT OF INTEREST}

The authors declare no conflict of interest.

\section{ACKNOWLEDGEMENTS}

We thank participating patients and their family for being cooperative throughout the study. We also thank all colleagues who contributed to this work by providing their valuable advice on experimental methods.

\footnotetext{
1 de Vega S, Iwamoto T, Yamada Y: Fibulins: multiple roles in matrix structures and tissue functions. Cell Mol Life Sci 2009; 66: 1890-1902.

2 Segade F: Molecular evolution of the fibulins: implications on the functionality of the elastic fibulins. Gene, 464: 17-31.

3 Cooley MA, Kern CB, Fresco VM et al: Fibulin-1 is required for morphogenesis of neural crest-derived structures. Dev Biol 2008; 319: 336-345.

4 Debeer P, Schoenmakers EF, Twal WO et al: The fibulin-1 gene (FBLN1) is disrupted in a t(12;22) associated with a complex type of synpolydactyly. J Med Genet 2002; 39 98-104.

5 Appella E, Weber IT, Blasi F: Structure and function of epidermal growth factor-like regions in proteins. FEBS Lett 1988; 231: 1-4.
} 
6 Adzhubei IA, Schmidt S, Peshkin L et al: A method and server for predicting damaging missense mutations. Nat Meth 2010; 7: 248-249.

7 Hucthagowder V, Sausgruber N, Kim KH, Angle B, Marmorstein LY, Urban Z: Fibulin-4: a novel gene for an autosomal recessive cutis laxa syndrome. Am J Hum Genet 2006; 78: $1075-1080$

8 Loeys B, Van Maldergem L, Mortier G et al: Homozygosity for a missense mutation in fibulin-5 (FBLN5) results in a severe form of cutis laxa. Hum Mol Genet 2002; 11 2113-2118.

9 Schultz DW, Klein ML, Humpert AJ et al: Analysis of the ARMD1 locus: evidence that a mutation in HEMICENTIN-1 is associated with age-related macular degeneration in a large family. Hum Mol Genet 2003; 12: 3315-3323.

10 Stone EM, Braun TA, Russell SR et al: Missense variations in the fibulin 5 gene and age-related macular degeneration. N Engl J Med 2004; 351: 346-353.
11 Stone EM, Lotery AJ, Munier FL et al: A single EFEMP1 mutation associated with both Malattia Leventinese and Doyne honeycomb retinal dystrophy. Nat Genet 1999; 22: 199-202.

12 Muriel JM, Dong C, Vogel BE: Distinct regions within fibulin-1D modulate interactions with hemicentin. Exp Cell Res 2012; 318: 2543-2547.

13 Tran H, VanDusen WJ, Argraves WS: The self-association and fibronectin-binding sites of fibulin-1 map to calcium-binding epidermal growth factor-like domains. J Biol Chem 1997; 272: 22600-22606.

14 Keshava Prasad TS, Goel R, Kandasamy K et al: Human protein reference database2009 update. Nucleic Acids Res 2009; 37: D767-D772.

15 Madej T, Addess KJ, Fong JH et al: MMDB: 3D structures and macromolecular interactions. Nucleic Acids Res 2012; 40: D461-D464.

16 Lu HS, Chai JJ, Li M, Huang BR, He CH, Bi RC: Crystal structure of human epidermal growth factor and its dimerization. J Biol Chem 2001; 276: 34913-34917. 